Conclusions: This study has shown that Nedd4 and Kcnal, genes encoding proteins either forming ion channels or modulating their activity, showed dysregulation following treatment with antipsychotics, which may provide important clues to the pathogenesis of schizophrenia.

\section{The Australian Biomarkers Lifestyle and Imaging flagship study of ageing}

\section{K Ellis' ${ }^{1}$ D Ames' ${ }^{1}$, R Martins², P Hudson ${ }^{3}$, C Masters ${ }^{1}$}

'The University of Melbourne, Melbourne, Australia; ${ }^{2}$ Edith Cowan University; and ${ }^{3} \mathrm{CSIRO}$, Australia

Background: The potential to optimize treatment and preventative strategies in the delay and prevention of Alzheimer's disease (AD) relies in part on the capacity to make early diagnoses and monitor disease progression. The Australian Biomarkers Lifestyle and Imaging (AIBL) study is a 3-year longitudinal cohort study that aims to improve understanding of the pathogenesis and diagnosis of AD using neuropsychological, neuroimaging and biomarker techniques, and to examine lifestyle and dietary factors associated with $\mathrm{AD}$ and healthy aging.

Methods: A total of 1000 volunteers (minimum age 65 years) were recruited, comprising 200 participants from the following groups: 1) AD, 2) mild cognitive impairments, 3) healthy volunteers (ApoE4+), 4) healthy volunteers (ApoE4-) and 5) 'memory complainers' (ie healthy volunteers reporting subjective memory complaints). At baseline and 18 months, all participants received a clinical/neuropsychological assessment and blood biomarker analysis, with a subgroup also receiving [C-11]PIB-PET and magnetic resonance imaging scans. Participants also completed questionnaires assessing diet and exercise patterns, with a subgroup receiving actigraph accelerometer measurement of activity levels and dual-energy X-ray absorptiometry measures of body composition.

Results: Patterns of change in individual measures (neuropsychology, neuroimaging and biomarkers) were examined within each population group. Changes in neuropsychological measures were correlated with neuroimaging and biomarker measures to establish convergent validity.

Conclusions: This forms the largest study of its kind ever undertaken in Australia. The current study identified neuroimaging, biomarker and neuropsychological measurements of longitudinal changes in a large cohort and enhanced knowledge of lifestyle and dietary factors associated with AD and healthy aging.

\section{Clozapine - fatal constipation more common than fatal agranulocytosis}

\author{
P Ellis', M Harrison-Woolrych², R McLean²
}

'Department of Psychological Medicine, Wellington School of Medicine, University of Otago; and ${ }^{2}$ Department Preventative and Social Medicine, University of Otago, New Zealand

Background: Premarketing evaluation of side-effects of medication is too small to evaluate rarer side-effects. This requires effective postmarketing pharmacovigilance. While spontaneous reporting to a national center is encouraged, more active methods are required to recruit larger cohorts of known size so rates of rare adverse events can be estimated.

Methods: The New Zealand Intensive Medicines Monitoring Programme (IMMP) prospectively examines the safety of marketed medicines using prescriptionevent monitoring methodology. Cohorts of patients are established using prescription data from pharmacies throughout the country. The IMMP obtains reports of adverse events from multiple sources, including from follow-up questionnaires sent to patients' doctors, spontaneous reports from health professionals, pharmaceutical company reports and linkage to national mortality and morbidity databases. An evaluation of atypical antipsychotics using this approach indicated high levels of GI side-effects with clozapine.

Results: A large number of cases of constipation were identified, some severe. Two subjects suffered toxic megacolon, one paralytic ileus, one bowel ischemia requiring resection and one bowel perforation. The latter two subjects died of complications of surgery. Two other subjects were shown to suffer esophageal dysmotility, one requiring surgical intervention.

Conclusions: Clozapine interacts with a range of muscarinic, serotonergic and other receptors to have particularly marked effects on the GI tract. These effects are predictable and can be managed provided adequate inquiry is made into symptoms. Only one New Zealander had died of clozapine agranulocytosis - at least three had died of consequences of constipation.

\section{Age differences in mental health literacy}

\section{Farrer', H Christensen', LS Leach', KM Griffiths', AF Jorm ${ }^{2}$}

'Centre for Mental Health Research, The Australian National University, Canberra, Australia; and ${ }^{2} \mathrm{ORYGEN}$ Research Centre, The University of Melbourne, Melbourne, Australia

Background: The community's understanding of mental health problems, their risk factors, treatments and sources of help may vary as a function of age. 